\title{
Low Energy Microanalysis by EDS or WDS? Comparisons and Concerns from an Analytical Services Laboratory Perspective
}

\author{
Craig S. Schwandt ${ }^{1}$ \\ ${ }^{1}$ McCrone Associates, Inc. 850 Pasquinelli Drive, Westmont, IL 60559 USA
}

The first decade of the twenty-first century witnessed some fantastic improvements in electron optics and detectors. The intense coherent electron beams of not just field emission gun (FEG) instruments but also of tungsten (W) filament instruments have pushed the resolving power easily into the sub-micrometer scale and into the nanometer scale for FEG. Concurrent improvements in backscattered electron detection routinely reveal the heterogeneity of materials, many of which were previously considered homogeneous. Although the beam diameter of FEG instruments is easily two to nearly three orders of magnitude narrower than twentieth century $\mathrm{W}$ gun instruments, the electron beam energy is a key factor to insuring that the interaction volume, and source of x-rays, created by interaction of the sample with a sustained electron beam, is placed in the volume of interest. The analyst can adjust the electron beam energy to control the depth and size of the interaction volume in the sample, by balancing it against the average atomic mass, and therefore electron beam stopping power of the sample. For example one can model the range of the interaction volume [1]. Using silicon as an example the range of interaction volume is about 0.5 micrometers at $5 \mathrm{kV}$, whereas it is about 3.0 micrometers at $15 \mathrm{kV}$.

Most clients of analytical service laboratories are these days interested in the major element composition of aggregate components of heterogeneous mixtures, or of individual particulate. Analysis of bulk homogeneous solid materials, at least at the scale of the measurements, is a much less frequent request. Therefore there is great interest in using low energy microanalysis to aid in reducing the interaction volume to a size similar to the volume of interest in the sample.

The increased low energy sensitivity of the latest generation of silicon drift detectors (SDD) requires consideration for quantitative micro and nanoanalysis. The increased ease of use of new energy dispersive spectrometer (EDS) system software also allows the analyst to measure and create their own standards database to use for quantification, which if matrix matched standards are used, reduces the uncertainties associated with the matrix corrections. The acquisition speed of SDD systems makes them attractive as one can more quickly achieve peak to background count statistics that potentially lower the detection limits [2]. Although EDS acquisition is fast, with an energy resolution of approximately 130 $\mathrm{eV}$, can it really be as useful relative to a wavelength dispersive (WDS) electron microprobe, with energy resolutions of about $10 \mathrm{eV}$ for many elements? What is the detector response of WDS systems like for delicate low energy analysis? How do the total acquisition times compare?

Provided that one is concerned primarily with essential structural constituent elements of a material, otherwise considered major elements, then the results are promising depending on the elements of interest present. Limited examples are discussed here and additional examples will be discussed at the conference, especially those that may have interference issues of low energy lines with oxygen and carbon. Additionally, the analyst can increase the confidence level of the quantitative results if they constrain the stoichiometry of the solution by crystal structure determination through the use of a method such as electron backscatter diffraction. 
Consider the comparison of approaches for the mineral zircon. The quantitative EDS results of low energy $5 \mathrm{kV}$ microanalysis are comparable to those at $15 \mathrm{kV}$. Examine the general peak to background ratios of the low energy EDS and WDS spectra.
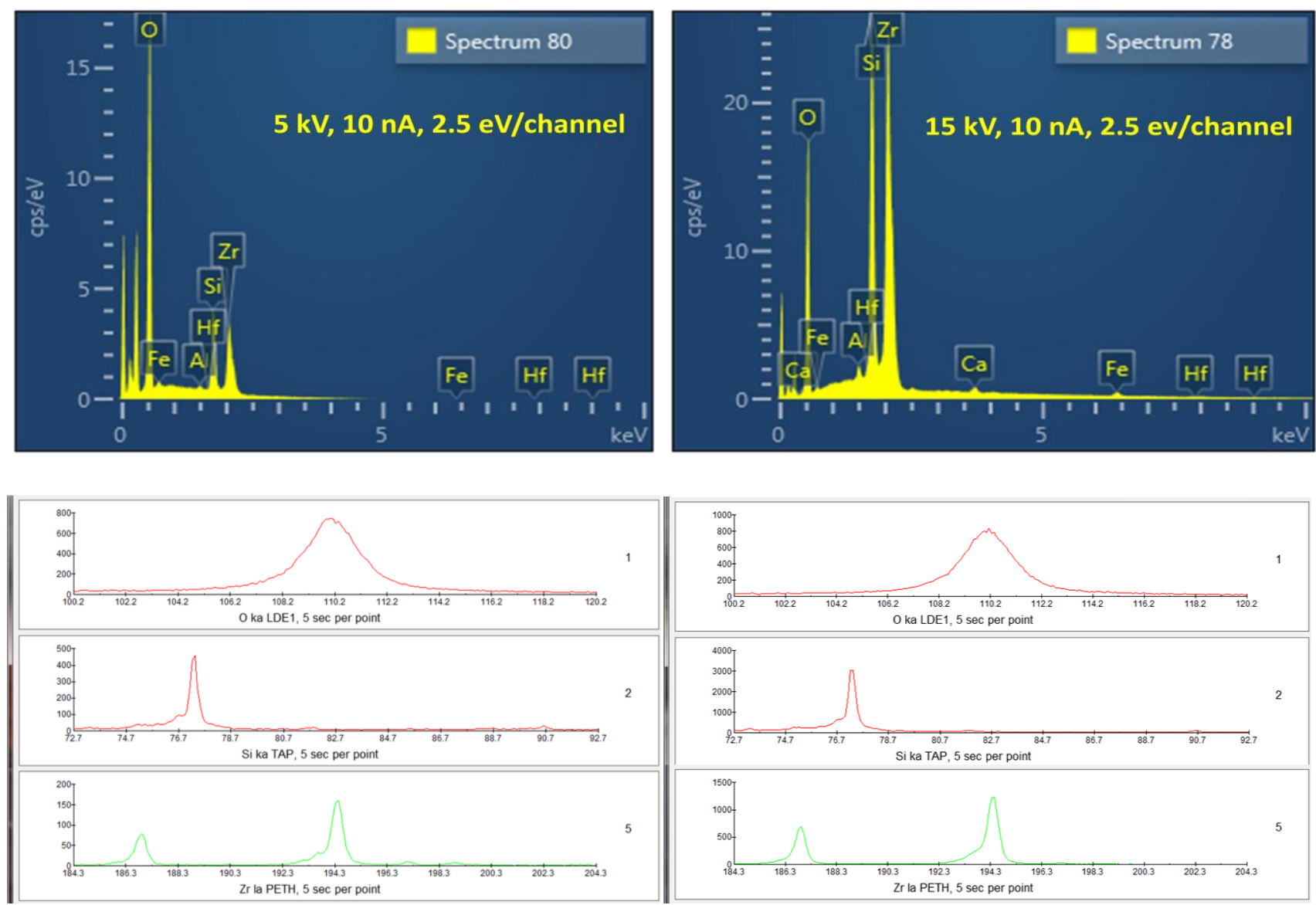

Obviously, the peak intensities are greater for high energy, but if one converts the scale of the WDS results by dividing by the beam current, $10 \mathrm{nA}$, then the WDS peak intensities are comparable to the EDS. Therefore, the WDS method is really only most useful in the case of minor or trace elements or for peak overlap instances. The practical speed, with which one can achieve reasonable characteristic quantitative results by EDS, can only be achieved by WDS for simple compounds such that each of the WDS spectrometers only needs to be used one time per analysis. Therefore, when considering the reduction in interaction volume, the use of low energy micro or nanoanalysis by EDS is a very pragmatic tool for analytical service laboratories.

\section{References}

[1] Goldstein, J., et. al., Scanning Electron Microscopy and X-ray Microanalysis: 3 Springer, 675p.

[2] D. Newbury and N. Ritchie, Microsc Microanal 15(Suppl 2) (2009) 6. 